theoretical determination of the steepness of the adiabatics in the plane $(p, v)$, it seems that the latter are'steeper than the former as regards inclination to the $v$-axis. Hence there is no point of maximum temperature on the adiabatic; but, on the other hand, there is a point of minimum temperature. This temperature for any given adiabatic, is that corresponding to the isothermal passing through the point of intersection of the adiabatic with the maximum.density curve. I have not altered the text above, however, as the remarks and figure may conceivably apply to some substance other than water.

The Theorems as far as Proposition 32, of the first book of Euclid's Elements, proved from First Principles.

$$
\text { By David Traile, M.A., B.Sc. }
$$

Proposition 4.

Given * $A B=D E, A C=D F, \angle A=\angle D$.

Suppose you start from B, and walk along BA a certain distance $a$ to $A$; then at $A$ you turn at a certain angle into another road $\mathbf{A C}$; then you walk along $\mathbf{A C}$ a certain distance $b$ to $\mathbf{C}$. Again you start from $\mathrm{E}$, walk a distance $a$ along $\mathrm{ED}$; turn off at D into DF at the same angle as before; then walk the distance $b$ along DF to F. Since you have gone through the same set of movements in the two cases, and since the same cause always produces the same result, $\dagger$ the results in the two cases must be the same, that is, you will arrive in both cases, at the same distance from the starting point. Hence $\mathbf{B C}=\mathbf{E F}$.

\title{
Proposition 5.
}

Given $A B=A C$.

From a certain point $A$, two lines $A B, A C$ are drawn. Two points $B, C$ equally distant from $A$ are joined. The same causes which determine the size of $\angle B$ also determine the size of $\angle C$. Hence $\angle \mathbf{B}=\angle \mathbf{C}$.

* For figures see Mackay's Elements of Euclid.

+ This axiom, ss well as its converse, is assumed in every Science. 
Given $\angle B=\angle C$.

\section{Proposition 6.}

From the two ends of a certain base line BC, two lines BA, CA are drawn making equal angles with $\mathrm{BC}$. The two lines meet at $\mathrm{A}$. The causes which determine the length of $A B$, also determine the length of $\mathrm{AC}$. Hence $\mathrm{AB}=\mathbf{A C}$.

\section{Proposition 8.}

Given $A B=D E, A C=D F, B C=E F$.

Suppose you start from $B$, and walk along $B A$ to $A$, then at $A$ you turn at a certain angle $A$ into $A C$, and walk along $A C$ to $C$. Again you start from $E$, and walk along $E D$ to $D$; then at $D$ you turn at a certain angle $D$ into $D F$, and walk along $D F$ to $F$. Now $\mathrm{BC}=\mathrm{EF}$, that is, the results of the two sets of movements are equal. Hence the causes must be equal. In the one case the causes are $\mathrm{BA}, \angle \mathrm{A}, \mathrm{AC}$; in the other $\mathrm{ED}, \angle \mathrm{D}, \mathrm{DF}$. But $\mathrm{BA}=\mathrm{ED}$, and $\mathrm{AC}=\mathrm{DF}$. Hence $\angle \mathrm{A}=\angle \mathrm{D}$.

\section{Propositions 13 and 14.}

These two Propositions follow directly from the Definitions of a right angle and a straight line.

Proposition 15.

Given $A B, C D$ two lines intersecting in $E$.

Since $A B$ bisects the infinite plane, and $C D$ bisects the same intinite plane; therefore the parts of the plane lying between these two lines are equal. Hence $\angle \mathbf{A E C}=\angle \mathrm{BED}$.

Propositions 16 and 17.

These two Propositions need not be considered as they are included in the 32nd.

\section{Proposition 18.}

Given $A C$ greater than $A B$.

$\angle B$ cannot be equal to $\angle C$; for if it were the effect would be that $\mathrm{AB}$ would be equal to $\mathrm{AC}$. Hence $\angle \mathrm{B}$ must either be greater or less than $\angle \mathrm{C}$. Now, suppose the smaller side $A B$ gradually to diminish, $\angle B$ cannot, owing to this change in the length of $A B$, become equal to $\angle C$, but must still remain greater or less. $A s A B$ continuously diminishes, angles $\mathbf{B}$ and $C$, if they change, must do so 
continuously. Suppose $\mathrm{AB}$ at last to vanish, $\angle \mathrm{C}$ also vanishes, but not $\angle B$. Hence $\angle B$ must have been greater than $\angle C$ all along.

Proposition 19.

Given $\angle B$ greater than $\angle C$.

$A B$ cannot be equal to $A C$, therefore $A B$ is either greater or less than AC. Now, suppose $\angle B$ to remain constant, and $\angle \mathbf{C}$ to diminish gradually, $\mathbf{A B}$ will still remain either greater or less than $A C$; and when $\angle C$ vanishes $A B$ also vanishes, but not $A C$. Hence $A C$ must have been greater than $A B$ all along.

Proposition 20.

To prove $B A+A C$ greater than $B C$.

This is axiomatic, therefore no proof is necessary.

Proposition 21.

I. To prove $B A+A C$ greater than $B D+D C$.

This also is axiomatic.

The following proof, however, may be given :-

Join AD and produce it to meet BC in $E$.

Suppose BC to be an elastic cord with its ends fixed at B, C. Let it be displaced by the point of a rod which runs along EB. The result of this continuous displacement must be either continuous increase or continuous decrease, but the displacement to $D$ has caused an increase in the length of $\mathrm{BC}$, therefore the further displacement to $A$ must cause further increase. Hence $\mathrm{BA}+\mathrm{AC}$ is greater than $B D+D C$.

II. To prove $\angle B D C$ greater than $\angle B A C$.

Let us now consider the effect of the continuous displacement of $\mathrm{BC}$ on the size of the contrined angle. At $\mathbf{E}$ the contained angle= two right angles. At $D$ the contained angle $B D C$ is less than two right angles, therefore any further displacement means further decrease in the angle. Hence $\angle \mathrm{BDC}$ is greater than $\angle \mathrm{BAC}$.

\section{Proposition 24.}

Given $A B=D E, A C=D F, \angle A$ greater than $\angle D$.

$\mathrm{BC}$ is not equal to $\mathrm{EF}$, therefore $\mathrm{BC}$ is either greater or less than EF. Now, suppose $\angle A$ to increase and $\angle D$ to diminish, BO will still remain greater or less than EF. If $\angle A$ become a straight angle, then $B C=A B+A C$, and if $\angle D$ vanish, then $E F=$ the differ- 
ence between $\mathrm{ED}$ and DF. Hence $\mathrm{BC}$ is now greater than $\mathrm{EF}$, and must have been greater all along.

Proposition 25.

Given $A B=D E, A C=D F, B C$ greater than $E F$.

When $\mathrm{BC}$ is the greatest possible, that is, when $\mathrm{BC}=\mathrm{BA}+\mathrm{AC}$, then $\angle A=2$ right angles; and when $E F$ is the least possible, that is, when $\mathbf{E F}=$ the difference between $\mathrm{ED}$ and $\mathrm{DF}$, then $\angle \mathrm{D}$ vanishes. Hence when $\mathbf{B C}$ is greater than EF, then also is $\angle A$ greater than $\angle D$.

Proposition 26.

Given $\angle B=\angle E, \angle C=\angle F, B C=E F$.

In the one triangle any base line $B C$ has been taken, and at its ends two angles $\mathrm{B}, \mathrm{C}$ are formed by the two lines $\mathrm{BA}, \mathrm{CA}$, which meet at $A$; in the other triangle another base line $\mathrm{EF}$, equal to $\mathrm{BC}$, has been taken, and two angles, E, F respectively equal to $\mathrm{B}, \mathrm{C}$, are formed by the two lines $\mathrm{ED}, \mathrm{FD}$, which meet at $\mathrm{D}$. We have the same causes in both cases, hence the results must be the same; that is, $\mathrm{BA}=\mathrm{ED}, \mathrm{AC}=\mathrm{DF}$.

Parallel Lines.

Definition :-Parallel lines are lines running in the same direction. Axiom:-Through the same point two parallel lines cannot be drawn.

Lomma:-Parallel lines never meet. For if they did, then through the same point there could be drawn two parallel lines which is impossible.

Proposition 27.

Given $\angle A G H=\angle G H D$, or $\angle B G H=\angle G H C$.

First Proof. From the ends of the base line GH there are certain angles laid off on the one side, and also equal angles on the other; if the effect on the one side of GH is that the lines meet, then they must also meet on the other, which is impossible.

Second Proof. Suppose CD to revolve anti-clockwise round $\mathbf{H}$ through the angle $\mathrm{DHG}$, then round $\mathrm{G}$ clockwise into the position AB. If, in its new position $A B$, it is not parallel to its original one, but meets it in a certain point, we should have a line after two revolutions equal and opposite, inclined at an angle to its original position, which is impossible. 


\section{Proposition 28.}

I. Given $\angle E G B=\angle G H D$ or $\angle F H C=\angle H G A$.

If, because $\angle \mathrm{EGB}=\angle \mathrm{GHD}, \mathrm{AB}, \mathrm{CD}$ should meet towards $\mathrm{BD}$, then because $\angle \mathrm{FHC}=\angle \mathrm{HGA}, \mathrm{AB}, \mathrm{CD}$ must meet towards A, C, which is impossible.

II. Given $\angle A G H+\angle G H C=2$ right angles, or $\angle B G H+\angle G H D$ $=2$ right angles.

First Proof. As in I.

Second Proof. Similar to Second Proof of Proposition 27.

Proposition 29.

Given $A B$ parallel to $C D$.

I. $\angle \mathrm{AGH}=\angle \mathrm{GHD}$, by a proof similar to Second Proof of Proposition 27 .

II. $\angle \mathrm{BGH}+\angle \mathrm{GHD}=2$ right angles in the same way.

III. To prove $\angle \mathrm{EGB}=\angle \mathrm{GHD}$.

First Proof. The size of $\angle$ EGB depends on two causes, the directions of the two lines GE, GB. The size of $\angle$ GHD depends on two causes, the directions of the two lines $\mathrm{HG}, \mathrm{HD}$; but the directions of $\mathrm{GE}, \mathrm{HG}$ are the same, and also of $\mathrm{GB}, \mathrm{HD}$, because they are parallel. Hence $\angle \mathrm{EGB}=\angle \mathrm{GHD}$.

Second Proof. There is nothing given as to the length of GH. Every proof, then, must be independent of the length of GH. Suppose GH gradually to diminish, and at last to vanish. Now when $G H$ vanishes, that is when $G, H$ coincide, then also $A B, C D$, since they are parallel, will coincide; for if they did not we should have two parallel lines drawn through the same point. Hence $A B$, $\mathrm{CD}$ coincide. Hence also $\angle \mathrm{EGB}$ and $\angle \mathrm{GHD}$ coincide and are equal.

\section{Proposition 32.}

Hamilton's Proof.* Suppose BC to revolve round B through $\angle B$, till it coincides with $B A$, then round $A$ through $\angle A$ till it reaches the position $\mathrm{AC}$, then round $\mathrm{C}$ through $\angle \mathrm{C}$, till it returns to its original position, but with ends inverted. $\mathrm{BC}$ must have revolved through two right angles. Hence $\angle A+\angle B+\angle C=2$ right angles.

Professor Henrici in Nature, 13th March 1884, objects to this

* See Casey's Elements of Euclid, pp. 244-246. The same proof, sub. stantially, occurs in Playfair's edition of Simson's Euclid. 
proof, on the ground that in the same way the angles of a spherical triangle might be proved equal to two right angles. On the contrary, a similar mode of proof will show that the angles of a spherical triangle are greater than two right angles. For we must now consider the revolution of planes containing the great circles of which the sides of the spherical triangle are arcs. Suppose, then, a plane by revolving to coincide in turn with the three sides of a spherical triangle. This plane in its three positions has always one point common, that is the centre of the sphere. The result of the three revolutions through the three spherical angles, is that the plane coincides with its original position, but with ends reversed. Now a plane can thus reverse its position by turning through two right angles, only on condition that it remains, during the revolution, perpendicular to the same fixed plane, that is that its axis of revolution is not subjected to tilting. Now, this is a condition that cannot be satisfied by a plane which coincides in turn with the three sides of a spherical triangle (except in the case when one side vanishes). Hence the three angles of a spherical triangle are greater than two right angles.

Ninth Meeting, July 11th, 1884.

Dr R. M. Ferguson in the Chair.

\section{Application of the Multiplication of Matrices to prove a Theorem in Spherical Geometry.}

By Professor Chrystal, University of Edinburgh.

The theorem in question is that if two of the diagonals of a spherical quadrilateral be quadrantal arcs, the third diagonal is also a quadrantal arc. (Fig. 31.)

Denote the direction cosines of the radius to the point 1 by $l_{1}, m_{1}, n_{1}$, dc., and $l_{1} l_{2}+m_{1} m_{2}+m_{1} n_{2}$ by 12 .

Then our conditions give $12=0,34=0$, and we have to prove $56=0$.

The equation to the plane 13 is 